Somayeh RAHMANI, PhD Candidate

E-mail: somayehrahmani28@gmail.com

Department of Mathematics, Islamic Azad University

Qazvin Branch, Qazvin, Iran

Associate Professor Reza Kazemi MATIN,PhD

E-mail: rkmatin@kiau.ac.ir (Corresponding author)

Department of Mathematics, Islamic Azad University

Karaj Branch Karaj, Iran

Mohsen KHUNSIAVASH, PhD

E-mail:mfsiavash@gmail.com

Department of Mathematics, Islamic Azad University

Qazvin Branch, Qazvin, Iran

Zohreh MOGHADAS, PhD

E-mail:Zmoghaddas@qiau.ac.ir

Department of Mathematics, Islamic Azad University

Qazvin Branch, Qazvin, Iran

\title{
CROSS-EFFICIENCY EVALUATION IN DATA ENVELOPMENT ANALYSIS WITH STOCHASTIC DATA: A CHANCE- CONSTRAINED PROGRAMMING APPROACH
}

Abstract. Cross-efficiency evaluation in Data envelopment analysis (DEA) has been accepted as a useful tool for performance evaluation and ranking of decision making units. However, the non-uniqueness of optimal weights in this evaluation has reduced the usefulness of this powerful method. As a result, it is recommended that secondary goals be introduced in cross-efficiency evaluation. Another issue in applying cross-efficiency DEA models is considering uncertainty in input and output variables. In this paper, the cross-efficiency model is modified to deal with stochastic data by applying chance-constrained approach. Some new stochastic DEA models and their associated deterministic versions are introduced. Furthermore, we discussed the existence of multiple weights in cross-efficiency method in the presence of stochastic data. As the result, we suggest some new secondary goals to better discriminate DMUs and obtain optimistic and pessimistic efficiency scores. Some numerical examples are used for illustration purposes.

Keywords: Cross-efficiency method; Secondary goals; Stochastic crossefficiency; Multiple weights.

JEL Classification: C61, C67 


\section{Introduction}

Data envelopment analysis (DEA) was introduced by Charnes et al. (1978). DEA belongs to non-parametric group of ways to assess the efficiency of decisionmaking units (DMUs) with several inputs and outputs.Also Charneset al.(1978)presented a model for assessing DMUs called CCR model which is based on the assumption of constant returns to scale.Taking the DMU under evaluation, DEA maximizes the weight of all inputs over the weight of all outputs so that the ratio of all units cannot be greater than 1 . All evaluations would result from the data of inputs and outputs( $\mathrm{Li}$ et al., 2015). Nowadays, the use of DEA technique has been rapidly expanding to evaluate different organizations and industries such as banking, energy, health care and etc. (Chen et al.,2016; Mardani et al.,2016).See (Emrouznejad et al., 2018) for more details.

Cross-efficiency evaluation method was employed for the first time by Sexton et al.(1986) to address such concerns in DEA modeling by providing more flexible and realistic weighting approach which is called peer-evaluation method. Successful applications of cross-efficiency in DEA literature shows the advantage of applying this method in the applications. The idea of using peer-evaluation instead of self-evaluation in cross-efficiency method provided an extension to the theory of DEA by promoting the power of individual voices in the process peerevaluation,(Lim et al., 2014). This method is then improved in Doyle and Green (1994). Efficiency among DMUs with weight schemes was introduced by Anderson et al.(2002).

In spite of merits of DEA cross-efficiency evaluation and its wide applications, it still has some short falls. For each DMU, the fact that DEA optimal weights are not unique may reduce the usefulness of cross-efficiency evaluation (Sexton et al. 1986). As Doyle and Green (1994) mentioned, multiple optimal weights acquired from classic DEA models can be used as secondary goals for better discrimination among DMUs. Evaluation of DEA cross-efficiency on the basis of Pareto improvement model was proposed by $\mathrm{Wu}$ et al. (2016). During recent years, the research concerning cross-efficiency evaluation has developed fast. Some of the significant studies in this field are as follows: Javier Alcaraz et al. (2013), Oral et al. (2015), Soltanifar et al.(2013), Li et al. (2018).

In majority of conventional DEA models, a basic assumption is that the input and output data of decision-making units (DMUs) are known certainty. However, there are many cases where only uncertain data are available. Promising attempts have recently been made to address randomness in data either stochastic input or stochastic output in DEA model. Cooper et al.(2004) extended DEA model through the use of chance constrained programming formulations to treat congestion in DEA through dealing with all inputs and outputs with random variations. A key feature of his study is that stochastic variables are considered in DEA method and stochastic DEA model was formulated into a deterministic equivalence. As a result of this formulation, stochastic DEA can be solved with any business computer software, including any secondary programming required. The deterministic equivalents could then be transformed to quadratic programming 
Cross-efficiency Evaluation in Data Envelopment Analysis with Stochastic Data: A Chance-constrained Programming Approach

models, which are non-linear programs. A model must have enough flexibility to take account of the potential uncertainty in outputs and inputs and yield more compelling results. Therefore, an introduced stochastic model to the proposed model improved the outputs. Insignificant effort has been made for a stochastic chance constrained input and output in cross-efficiency model. In this study, we examine cross-efficiency with stochastic input and output and discuss some of the secondary goals. Kuosmanen and Johnson (2010)improved similar semi-parametric DEA model. Kuosmanen and Kortelainen(2012)and Kuosmanen et al.(2015), and Kao and Liu (2009) used stochastic DEA technique to treat the stochastic data. Considering stochastic DEA technique in efficiency measurement gathered in (Khodabakhshi 2009; Hiroshi and Seiford 1999), Azadi and Saen(2011) suggested the chance-constrained DEA model that enables the stochastic approach in all data variables to specify the most suitable third-party reverse logistics to optimize the decision making process.

In this papers, the topic of cross-efficiency DEA model was treated using multiple weights by considering stochastic data. Stochastic characterizations of cross-efficiency scores in DEA use of chance-constrained approach to deal with stochastic variation in constraints so as to change them to nonlinear counterparts. The developed algorithm is based on the basic CCR model by considering stochastic environment under assumptions that all inputs and outputs data are independent random variables and normally distributed. We considered presence of multiple optimal weights in the presence of stochastic data. Then, some new secondary goals are introduced for evaluating optimistic and pessimistic efficiency scores for any observed unit.

The remainder of the current research is organized as follows: The conventional cross-efficiency model is briefly reviewed in the Section 2. In Section 3 , the stochastic version of the cross-efficiency DEA model is proposed for the first time. The existence of the multiple weights is examined and explained in Section 4. Section 5 dedicated to introduce new secondary goals for providing the optimistic and pessimistic models in the presence of stochastic data. An illustrative example is presented in Section 6. Section 7 discusses the conclusions.

\section{Cross-Efficiency Model}

Suppose a number of $\mathrm{n}$ decision making units $\left(D M U_{j}, j=1 . \ldots . n\right)$ each of which use multiple inputs to produce multiple outputs. Note that the $\mathrm{i}^{\text {th }}$ input and $r^{\text {th }}$ output of each $D M U_{j}$ is mentioned as $x_{i j}(i=1, \ldots, m)$ and $y_{r j}(j=1, \ldots, s)$, respectively. Cross-efficiency method is generally calculated in two phases. The first phase is carried out using of the CCR model of Charnes et al.(1978) which can be displayed in its output oriented form as follows (1):

$$
\begin{gathered}
\max E_{d d}=\frac{\sum_{r=1}^{s} u_{r d} y_{r d}}{\sum_{i=1}^{m} v_{i d} x_{i d}} \\
\text { s.t } E_{d j}=\frac{\sum_{r=1}^{s} u_{r d} y_{r j}}{\sum_{i=1}^{m} v_{i d} x_{i j}} \leq 1 j=1,2, \ldots, n
\end{gathered}
$$


Somayeh Rahmani, Reza Kazemi Matin, Mohsen Khunsiavash ,Zohreh Moghadas

$$
u_{r d} \geq 0 \quad r=1, \ldots, s, v_{i d} \geq 0 \quad i=1, \ldots, m
$$

Where $v_{i d}(i=1 \ldots . m)$ and $u_{r d}(r=1 \ldots . . s)$ represent the $\mathrm{i}^{\text {th }}$ input and $r^{\text {th }}$ output weights of the under assessment unit $D M U_{d}$.

In the second stage of the method, the cross-efficiency score of $D M U_{j}$, by considering the inputs and outputs optimal weights obtained for evaluation of $D M U_{d}$ in model (1) is given by the following equation:

$E_{d j}=\frac{\sum_{r=1}^{S} u_{r d}^{*} y_{r j}}{\sum_{i=1}^{m} v^{*}{ }_{i d} x_{i j}} \quad d, j=1, \ldots, n$

Finally, for each $\operatorname{DMU}_{j}(j=1,2, \ldots, n)$ the average of all $E_{d j}(j=1,2,3, \ldots, n)$ is computed as follows:

$\overline{E_{J}}=\frac{1}{n} \sum_{d=1}^{n} E_{d j}(j 1,2, \ldots, n)$

$\bar{E}_{j}$ denotes the cross-efficiency score for $\operatorname{DMU}_{j}(j=1,2, \ldots, n)$. Note that each $E_{d j}$ is called a cross-efficiency value and also the defined average in Equation (3) in the DEA literature is used for ranking DMUs. Generally speaking, cross-efficiency defined average in Equation (3), not the individual scores defined in Equation (2).

As Model (1) is non-linear thus considering Charnes and Cooper's transformation (1984), we will arrive to the following linear model:

$\operatorname{Max} E_{d d}=\sum_{r=1}^{s} u_{r d} y_{r d}$

s.t. $\sum_{i=1}^{m} v_{i d} x_{i d}=1$

$\sum_{r=1}^{s} u_{r d} y_{r j}-\sum_{i=1}^{m} v_{i d} x_{i j} \leq 0 \quad j=1, \ldots, n$

$$
u_{r d}, v_{i d} \geq 0
$$

Note that cross-efficiency scores mentioned from (3), is the average of $E_{d j}$ and is not greater than one. In accordance to Doyle and Green (1994), here we elaborate on the notion of cross-efficiency according to the matrix mentioned in Table 1. $E_{d j}$ Is the cross-efficiency value of each $D M U_{j}(j=1,2 \ldots, n)$ based on a set of optimal weights obtained for DEA model while $D M U_{d}$ is being assessed (selfassessing). 
Cross-efficiency Evaluation in Data Envelopment Analysis with Stochastic Data: A Chance-constrained Programming Approach

In accordance to the cross efficiency method, while $D M U_{d}$ is being evaluated, the model searches feasible weights for the best efficiency score for this unit. Note that $E_{d d}$ (in the leading diagonal) is the average of the efficiency scores of each DMU $j$ while given the optimal weights of $D M U_{d}$. Here $E_{d d}$ is mentioned by $\bar{E}_{J}$. We argue that in Doyle and Green (1994), the efficiency score for $D M U_{d}$ is not taken into account as part of the average. Clearly, $E_{d j}(d \neq j)$ and $\bar{E}_{j}$ are not unique, as a result of the ever-present multiple optimal DEA weights in this model (4). For example, as multiple optimal weights can occur in DEA models, the crossefficiency concept may be judged as an unreliable method. Bear in mind that the above discussion output orientation and thus considering the same way with the output-oriented model, it is also possible to calculate the cross-efficiency as well.

Table 1:Cross-efficiency matrix

\begin{tabular}{|c|c|c|c|c|c|}
\hline DMU & $D M U_{1}$ & $D M U_{2}$ & ... & $D M U_{n}$ & Average \\
\hline$D M U_{1}$ & $\mathrm{E}_{11}$ & $\mathrm{E}_{12}$ & $\ldots$ & $\mathrm{E}_{1 n}$ & $\frac{1}{n} \sum_{o=1}^{n} \mathrm{E}_{1 o}$ \\
\hline$D M U_{2}$ & $E_{21}$ & $E_{22}$ & $\cdots$ & $\mathrm{E}_{2 n}$ & $\frac{1}{n} \sum_{o=1}^{n} \mathrm{E}_{2 o}$ \\
\hline$\cdots$ & $\ldots$ & $\cdots$ & $\cdots$ & $\cdots$ & $\ldots$ \\
\hline$D M U_{n}$ & $E_{n 1}$ & $\mathrm{E}_{n 2}$ & $\cdots$ & $\mathrm{E}_{n n}$ & $\frac{1}{n} \sum_{o=1}^{n} \mathrm{E}_{n o}$ \\
\hline
\end{tabular}

\section{Stochastic Cross-Efficiency}

In the presence of stochastic data, a DMU which is evaluated to perform efficiently may turn to be inefficient if such stochastic variations are taken into consideration. For better analyzing the real world problems (than what have been carried out in the standard DEA models), A number of researchers introduced stochastic input and output into the DEA model. (See Cooper et al. 2004;Cooper et al., 2002;Khodabakhshi et al., 2009)among others.

Following the notations introduced by Cooper et al. (2004), we use $\tilde{x}_{j}=$ $\left(\tilde{x}_{1 j}, \ldots, \tilde{x}_{m j}\right)^{t}$ and $\tilde{y}_{j}=\left(\tilde{y}_{1 j}, \ldots, \tilde{y}_{s j}\right)^{t}$ to represent random input and output vectors, respectively. It is assumed that inputs and outputs to be random variables with a multivariate normal distribution. The stochastic version of CCR envelopment model is formulated as follow; Cooper et al. (2004):

$$
\begin{array}{ll}
\max \varphi= & \sum_{r=1}^{s} u_{r d} \tilde{y}_{r d} \\
\text { s.t. } & \sum_{r=1}^{s} v_{i d} \tilde{x}_{i d}=1
\end{array}
$$




$$
\begin{aligned}
& p\left\{\sum_{r=1}^{s} u_{r d} \tilde{y}_{r j}-\sum_{i=1}^{m} v_{i d} \tilde{x}_{i j} \leq 0\right\} \geq 1-\alpha, \quad j=1, \ldots, n \\
& u_{r d} \quad \begin{array}{l}
v_{i d} \geq 0, \quad r=1, \ldots, s, \\
i=1, \ldots, m
\end{array}
\end{aligned}
$$

Where $\alpha$ is a previously specified value between 0 and 1 , which indicates the significance level of constraints and $p$ shows the probability and " $~$ " presents the data as random variables with a normal distribution.

The chance constrained Model (5) can be converted into its equivalent deterministic counterpart as follows; (see the appendix):

$$
\begin{aligned}
& \max \varphi=\sum_{r=1}^{s} u_{r d} y_{r d} \\
& \text { s.t. } \quad \sum_{i=1}^{m} v_{i d} x_{i d}=1 \\
& \sum_{r=1}^{s} u_{r d} y_{r j}-\sum_{i=1}^{m} v_{i d} x_{i j}-\Phi^{-1}(\alpha) \delta_{j} \leq 0, \forall j \\
& u_{r d}, v_{i d} \geq 0 \quad r=1, \ldots, s, i=1, \ldots, m \\
& \delta_{j} \quad \sum_{r=1}^{s} \sum_{t=1}^{s} u_{r} u_{t} \operatorname{Cov}\left(\tilde{y}_{r j}, \tilde{y}_{t j}\right)+\sum_{i=1}^{m} \sum_{l=1}^{m} v_{i} v_{l} \operatorname{Cov}\left(\tilde{x}_{i j}, \tilde{x}_{l j}\right) \\
& +2 \sum_{r=1}^{m} \sum_{i=1}^{m} u_{r} v_{i} \operatorname{Cov}\left(\tilde{y}_{r j}, \tilde{x}_{i j}\right), j, 1, \ldots, n
\end{aligned}
$$

Here, $\Phi$, the cumulative distribution is function of a standard normalrandom variable and $\Phi^{-1}$ is its inverse. $\operatorname{Cov}\left(\tilde{x}_{i j}, \tilde{x}_{l j}\right)$ And $\operatorname{Cov}\left(\tilde{y}_{r j}, \tilde{y}_{t j}\right)$ refer to variance of inputs and outputs, respectively. (See the Appendix) Now, based on (6), we can compute stochastic cross-efficiency scores. Note that if $\alpha=0.5$. is a predetermined level of probability, then $\Phi^{-1}(\alpha)=0$ and optimal value for $u_{r}^{*}, v_{i}^{*}$ in the stochastic version can be derived from solving the CCR model in which the mean values of inputs and outputs are employed (Wu et al. 2012).

\section{Definition of Stochastic Efficiency:}

A DMU assessed by Model (5) is stochastically efficient if and only if the following conditions are both satisfied, (Cooper et al. 2004):

(i) $\varphi_{0}^{*}=1$

(ii) $u^{*}, v^{*} \geq 0$ For all optimal solutions.

Example1. Consider six DMUs, each of them has two inputs and two outputs as reported in Table 2. The computed CCR scores, the matrix of cross-efficiency and a set of optimal weights are presented in Table 2. In Table 3 and 4 , the deterministic data listed in Table 2 are reported stochastically with variance of 0.01 and confidence interval of $95 \%$; i.e. $\alpha=0.05$ is used in (6). At first, the data are 
Cross-efficiency Evaluation in Data Envelopment Analysis with Stochastic Data: A Chance-constrained Programming Approach

selected stochastically (Table 3) and then according to the mentioned models, the cross-efficiency scores are presented in Table 4.

Table 2. Cross-efficiency scores with deterministic data for example 1

\begin{tabular}{|c|c|c|c|c|c|c|c|c|c|c|c|c|c|c|}
\hline \multicolumn{5}{|c|}{ Data } & \multicolumn{6}{|c|}{ Cross -Efficiency Scores } & \multicolumn{4}{|c|}{ Optimal Weights } \\
\hline$x_{1}$ & $x_{2}$ & $y_{1}$ & $y_{2}$ & DMU & (1) & (2) & (3) & (4) & (5) & (6) & $v_{1}^{*}$ & $v_{2}^{*}$ & $u_{1}^{*}$ & $u_{2}^{*}$ \\
\hline 1.5 & 0.2 & 1.4 & 0.35 & (1) & 1 & 0.42 & 1 & 0.49 & 0.51 & 0.47 & 0.52 & 1.12 & 0.68 & 0.12 \\
\hline 4 & 0.7 & 1.4 & 2.1 & (2) & 1 & 1 & 0.83 & 1 & 0.98 & 0.87 & 0.14 & 0.64 & 0.14 & 0.38 \\
\hline 3.2 & 1.2 & 4.2 & 1.05 & (3) & 0.71 & 0.34 & 1 & 0.53 & 0.52 & 0.4 & 0.31 & 0 & 0.22 & 0.06 \\
\hline 5.2 & 2 & 2.8 & 4.2 & (4) & 0.71 & 0.65 & 1 & 1 & 0.92 & 0.65 & 0.19 & 0 & 0.1 & 0.17 \\
\hline 3.5 & 1.2 & 1.9 & 2.5 & (5) & 1 & 1 & 0.83 & 1 & 0.97 & 0.87 & 0.11 & 0.51 & 0.11 & 0.3 \\
\hline 3.2 & 0.7 & 1.4 & 1.5 & (6) & 1 & 1 & 0.83 & 1 & 0.98 & 0.87 & 0.15 & 0.72 & 0.16 & 0.43 \\
\hline & & & & $\overline{E_{J}}$ & 0.9033 & 0.735 & 0.915 & 0.8367 & 0.8133 & 0.688 & & & & \\
\hline
\end{tabular}

Table 3. Stochastic data, $\alpha=0.05$

\begin{tabular}{|c|c|c|c|}
\hline \multicolumn{4}{|c|}{ Data } \\
\hline $\begin{array}{c}x_{1} \sim \\
N(\mu .001)\end{array}$ & $\begin{array}{c}x_{2} \sim \\
N(\mu . .001)\end{array}$ & $\begin{array}{c}y_{1} \sim \\
N(\mu .001)\end{array}$ & $\begin{array}{c}y_{2} \sim \\
N(\mu .001)\end{array}$ \\
\hline 1.5 & 0.2 & 1.4 & 0.35 \\
\hline 4 & 0.7 & 1.4 & 2.1 \\
\hline 3.2 & 1.2 & 4.2 & 1.05 \\
\hline 5.2 & 2 & 2.8 & 4.2 \\
\hline 3.5 & 1.2 & 1.9 & 2.5 \\
\hline 3.2 & 0.7 & 1.4 & 1.5 \\
\hline
\end{tabular}

Table 4. Stochastic cross efficiency scores and optimal weights

\begin{tabular}{|l|l|l|l|l|l|l|l|l|l|l|}
\hline \multicolumn{9}{|c}{ Cross-Efficiency Scores } & \multicolumn{5}{c|}{ Optimal Weights } \\
\hline $\mathbf{D M U}$ & $\mathbf{( 1 )}$ & $\mathbf{( 2 )}$ & $\mathbf{( 3 )}$ & $\mathbf{( 4 )}$ & $\mathbf{( 5 )}$ & $\mathbf{( 6 )}$ & $\boldsymbol{v}_{\mathbf{1}}^{*}$ & $\boldsymbol{v}_{\mathbf{2}}^{*}$ & $\boldsymbol{u}_{\mathbf{1}}^{*}$ & $\boldsymbol{u}_{\mathbf{2}}^{*}$ \\
\hline$(1)$ & 0.91 & 0.33 & 1.00 & 0.42 & 0.44 & 0.39 & 0.57 & 0.70 & 0.65 & 0.00 \\
\hline$(2)$ & 0.53 & 1.00 & 0.42 & 1.00 & 0.98 & 0.84 & 0.13 & 0.70 & 0.00 & 0.50 \\
\hline$(3)$ & 0.73 & 0.27 & 1.00 & 0.42 & 0.42 & 0.34 & 0.31 & 0.00 & 0.24 & 0.00 \\
\hline$(4)$ & 0.29 & 0.66 & 0.41 & 1.00 & 0.90 & 0.59 & 0.19 & 0.00 & 0.00 & 0.24 \\
\hline$(5)$ & 1.00 & 1.00 & 0.93 & 1.00 & 1.00 & 0.92 & 0.10 & 0.54 & 0.14 & 0.30 \\
\hline$(6)$ & 1.00 & 1.00 & 0.96 & 1.00 & 1.00 & 0.90 & 0.16 & 0.70 & 0.21 & 0.41 \\
\hline$\widetilde{\boldsymbol{E}_{J}}$ & $\mathbf{0 . 7 4}$ & $\mathbf{0 . 7 1}$ & $\mathbf{0 . 7 9}$ & $\mathbf{0 . 8 1}$ & $\mathbf{0 . 7 9}$ & $\mathbf{0 . 6 6}$ & & & & \\
\hline
\end{tabular}

Based on the results, the following ranking status in cross efficiency method is achieved for these data set:

- Deterministic data: $D M U_{3}>D M U_{1}>D M U_{4}>D M U_{5}>D M U_{2}>D M U_{6}$

- Stochastic data: $D M U_{4}>D M U_{3}=D M U_{5}>D M U_{1}>D M U_{2}>D M U_{6}$ 
Note that by allowing only $5 \%$ of unsatisfied constraints for model (4), the provided rank position is changed for some of the units. For example, $D M U_{4}$ achieved the best rank in stochastic case, while it was in the third place of performance in deterministic cross-efficiency evaluation. Only for $D M U_{2}$ and $D M U_{6}$ (two out of six), the stochastic rank is the same as deterministic rank. In the other word, for these units, we are almost sure that they are performing weak in comparison with the other units. For a complete analysis in ranking units based on stochastic cross-efficiency scores, different risk levels (i.e. different values for $\alpha$ ) should be used.

This simple example shows that if inputs and outputs are available as stochastic data, using stochastic cross-efficiency method provides useful information on relative performance of the units.

\section{Multiple Weights in Stochastic Cross-Efficiency Evaluation}

Due to occurrence of optimal multiple weights, the acquired crossefficiency scores are less likely to be unique. This is a significant weakness in the cross-efficiency model. To address this problem, Sexton et al.(1986)introduced the idea of secondary goals in the presence of the non- uniqueness of optimal weights in cross-efficiencies that was later developed by Doyle and Green (1994).

When the data are considered to be deterministic in cross-efficiency method, there may exist optimal multiple weights. All the obtained weights affect the cross-efficiency scores in (6). In this section, for the first time, we discussed the possibility of multiple optimal weights in the presence of stochastic. Moreover, we tried to verify if both stochastic data and optimal multiple weights affect the further definitions of cross efficiencies.

To answer this question, we chose a particular DMU from the example 1. Now consider the following deterministic optimization model:

$\max (\min ) \quad u_{1}$

s.t. $\quad \frac{\sum_{r=1}^{s} u_{r d} y_{r d}}{\sum_{i=1}^{m} v_{i d} x_{i d}}=E_{d d}$

$\frac{\sum_{r=1}^{S} u_{r d} y_{r j}}{\sum_{i=1}^{m} v_{i d} x_{i j}} \leq 1, \quad j=1, \ldots, n$

$$
u_{r d}, v_{i d} \geq 0, r=1, \ldots, s, i 1, \ldots, m
$$

The objective function of this model provides the feasible range for optimal weights of $u_{1}$; as the first output weight. The first equality constraint guarantees that CCR efficiency score of the under evaluation unit, i.e. $D M U_{d}$, unchanged. The other $\mathrm{e}=$ inequality constraints restrict the feasible region of input, output weights.

The stochastic counterpart of this model in general form is as follows:

$\max (\operatorname{minn}) \quad u_{1 d}$ 
Cross-efficiency Evaluation in Data Envelopment Analysis with Stochastic Data: A Chance-constrained Programming Approach

$$
\begin{aligned}
& \text { s.t } \sum_{r=1}^{s} u_{r d} y_{r d}-E_{d d} \sum_{i=1}^{m} v_{i d} x_{i d}=0 \\
& \sum_{r=1}^{s} u_{r d} y_{r j}-\sum_{i=1}^{m} v_{i d} x_{i j}-\Phi^{-1}(\alpha) \delta_{j} \leq 0, \forall j \\
& u_{r d}, v_{i d} \geq 0 \quad r=1, \ldots, s, i=1, \ldots, m \\
& \delta_{j}=\sum_{r=1}^{s} \sum_{k=1}^{s} u_{r} u_{k} \operatorname{Cov}\left(\tilde{y}_{r j}, \tilde{y}_{k j}\right)+\sum_{i=1}^{m} \sum_{l=1}^{m} v_{i} v_{l} \operatorname{Cov}\left(\tilde{x}_{i j}, \tilde{x}_{l j}\right) \\
& +2 \sum_{r=1}^{s} \sum_{i=1}^{m} u_{r} v_{i} \operatorname{Cov}\left(\tilde{y}_{r j}, \tilde{x}_{i j}\right), j=1, \ldots, n
\end{aligned}
$$

Consider the example1 mentioned in Section 3 and the associated Model (6). The achieved optimal inputs and outputs weights for $D M U_{2}$ are $\left(v_{1}^{*}, v_{2}^{*}\right)=(0.13,0.7)$ and $\left(u_{1}^{*}, u_{2}^{*}\right)=(0,0.5)$, respectively. Consider Table 5 , this unit has the same efficiency score considering Model (3.2) but it has different input and output weights; $\left(v_{1}^{*}, v_{2}^{*}\right)=(0,1.43)$ and $\left(u_{1}^{*}, u_{2}^{*}\right)=(0,0.48)$. $)$. In a similar manner, consider the computed result for $D M U_{6}$ by applying models (6) and (7) in Table 4. The input and output weights are $\left(v_{1}^{*}, v_{2}^{*}\right)=(0.16,0.7)$ and $\left(u_{1}^{*}, u_{2}^{*}\right)=(0.21,0.41)$. In Table 5 , the achieved optimal inputs and outputs weights for $D M U_{6}$ are $\left(v_{1}^{*}, v_{2}^{*}\right)=(0.14,0.77)$ and $\left(u_{1}^{*}, u_{2}^{*}\right)=(0.15,0.46)$, respectively. According to these results, the computed cross-efficiency score is ill-defined, even in the presence of stochastic data. As an instance, $D M U_{2}$ achieved two different cross-efficiency scores 0.71 and 0.87 in Table 4 and Table 5, respectively.

These examples clearly demonstrate the issue of existence multiple optimal weights for optimization model (6) in computing cross-efficiency scores. This issue has persuaded the researchers to introduce secondary goals to choose between optimal weights calculate cross-efficiency scores in discriminate data case.

\section{Table 5. Multiple optimal weights and cross-efficiency}

\begin{tabular}{|c|c|c|c|c|c|c|c|c|c|c|}
\hline \multicolumn{10}{|c|}{ Cross-Efficiency Scores } & \multicolumn{4}{c|}{ Optimal Weights } \\
\hline DMU & $\mathbf{( 1 )}$ & $\mathbf{( 2 )}$ & $\mathbf{( 3 )}$ & $\mathbf{( 4 )}$ & $\mathbf{( 5 )}$ & $\mathbf{( 6 )}$ & $\boldsymbol{v}_{\mathbf{1}}^{*}$ & $\boldsymbol{v}_{\mathbf{2}}^{*}$ & $\boldsymbol{u}_{\mathbf{1}}^{*}$ & $\boldsymbol{u}_{\mathbf{2}}^{*}$ \\
\hline $\mathbf{( 1 )}$ & 0.91 & 1.08 & 0.45 & 0.76 & 0.77 & 0.81 & {$[0,0.05]$} & {$[0,5]$} & {$[0,0.23]$} & {$[0,1.67]$} \\
\hline $\mathbf{( 2 )}$ & 0.58 & 1 & 0.29 & 0.7 & 0.69 & 0.71 & {$[0,0.05]$} & {$[0,1.43]$} & {$[0,0.05]$} & {$[0,0.48]$} \\
\hline $\mathbf{( 3 )}$ & 0.71 & 0.68 & 1 & 1 & 0.95 & 0.67 & {$[0,0.31]$} & {$[0,0.05]$} & {$[0,0.17]$} & {$[0,0.29]$} \\
\hline $\mathbf{( 4 )}$ & 0.29 & 0.65 & 0.41 & 1 & 0.88 & 0.58 & {$[0,0.19]$} & {$[0,0.05]$} & {$[0,0.05]$} & {$[0,0.24]$} \\
\hline $\mathbf{( 5 )}$ & 0.9 & 1.05 & 0.72 & 1.03 & 1 & 0.89 & {$[0,0.1]$} & {$[0,0.54]$} & {$[0,0.08]$} & {$[0,0.34]$} \\
\hline $\mathbf{( 6 )}$ & 0.99 & 1.05 & 0.79 & 1.02 & 1 & 0.9 & {$[0,0.14]$} & {$[0,0.77]$} & {$[0,0.15]$} & {$[0,0.46]$} \\
\hline$\widetilde{\boldsymbol{E}_{\mathbf{J}}}$ & $\mathbf{0 . 7 3}$ & $\mathbf{0 . 9 2}$ & $\mathbf{0 . 6 1}$ & $\mathbf{0 . 9 2}$ & $\mathbf{0 . 8 8}$ & $\mathbf{0 . 7 6}$ & & & & \\
\hline
\end{tabular}




\section{Optimistic and Pessimistic Models as Secondary Goals by Considering Stochastic Data}

Due to existence of multiple optimal weights in cross-efficiency evaluation, crossefficiency score may be ill-defined. Thus, as Doyle and Green (1994) claimed, a secondary goal could be taken into account to choose between alternative optimal weights to remove this deficiency. Doyle and Green (1994) proposed aggressive and benevolent secondary goals, which respectively minimize and maximize the efficiency of the virtual DMU while it is being compared to under evaluation observed DMU. The most prevalent secondary goal is utilizing the aggressive model which minimizes the efficiency score and chooses weights in a way that resulted in identification of the minimum efficiency of the aggregate DMU comprised of the remaining n-1 DMUs. The benevolent model has the same structure as aggressive model but it attempts to maximize the efficiency of the composite DMU.

In this section, aggressive and benevolent secondary goals in crossefficiency evaluation are extended for dealing with stochastic data. Therefore, we presented the results of considering the first example using either the aggressive method or the benevolent one with stochastic data. The proved example is then examined with the new proposed models.

In the aggressive model, the unit under evaluation minimizes aggregated cross-efficiencies of other units when it has the best weight as follows:

$$
\min (n-1) A_{k}=\sum_{j \neq k} \tilde{E}_{k j}=\sum_{j \neq k} \frac{\sum_{r} u_{r k} \tilde{y}_{r j}}{\sum_{i} v_{i k} \tilde{x}_{i j}}
$$

Taking the above expression as an objective function, the associated optimization model will a non-linear fractional, which cannot be easily solved. Instead of minimizing the fractional aggregate of cross-efficiency, Sexton et al.(1986) introduced a surrogate function, which subtracts the numerator from the denominator and minimizes the achieved function. The new aggressive model could be stated as follows:

$$
\begin{aligned}
\min & B_{k}=\sum_{j \neq k}\left(\sum_{r} u_{r k} \tilde{y}_{r j}-\sum_{i} v_{i k} \tilde{x}_{j}\right) \\
= & \sum_{r}\left(u_{r k} \sum_{j \neq k} \tilde{y}_{r j}\right)-\left(\sum_{i} v_{i j} \sum_{j \neq k} \tilde{x}_{i j}\right)
\end{aligned}
$$

Here, $\sum_{j \neq k} \tilde{y}_{r j}$ is the sum of stochastic outputs in which $D M U_{k}$ is excluded.Similarly, $\sum_{j \neq k} \tilde{x}_{i j}$ represents the combination of stochastic inputs of all DMUs except for $D M U_{k}$. In this formulation, the goal is to minimize the crossefficiency of DMUs as explained above. Note that the formulation in the benevolent form could be stated by maximizing this function.

So, the new proposed cross-efficiency evaluation with stochastic data has two phases: 
Cross-efficiency Evaluation in Data Envelopment Analysis with Stochastic Data: A Chance-constrained Programming Approach

(i) The optimization model (4) is solved for every observed unit. Based on the provided solutions $E_{k k}$ values are calculated by applying (2) fork $=$ $1, \ldots, n$.

(ii) $B_{k}$ As the aggressive secondary goal is achieved via solving the nonlinear programming model.

$\min \sum_{r=1}^{s} u_{r d} \sum_{j=1 . j \neq d}^{n} y_{r j}$

s.t. $\quad \sum_{i=1}^{m} v_{i d} \sum_{j=1 . j \neq d}^{n} x_{i j}=1$

$\sum_{r=1}^{s} u_{r d} y_{r d}-E_{d d}^{*} \sum_{i=1}^{m} v_{i d} x_{i d}=0$

$\sum_{r=1}^{s} u_{r d} y_{r j}-\sum_{i=1}^{m} v_{i d} x_{i j}-\Phi^{-1}(\alpha) \delta_{j} \leq 0, \forall j$

$u_{r d}, v_{i d} \geq \varepsilon \quad r=1, \ldots, s, i=1, \ldots, m$

$\delta_{j}=\sum_{r=1}^{s} \sum_{k=1}^{s} u_{r} u_{k} \operatorname{Cov}\left(\tilde{y}_{r j}, \tilde{y}_{k j}\right)+\sum_{i=1}^{m} \sum_{l=1}^{m} v_{i} v_{l} \operatorname{Cov}\left(\tilde{x}_{i j}, \tilde{x}_{l j}\right)$

$+2 \sum_{r=1}^{s} \sum_{i=1}^{m} u_{r} v_{i} \operatorname{Cov}\left(\tilde{y}_{r j}, \tilde{x}_{i j}\right), j=1, \ldots, n$

Where $\varepsilon>0$ is a non-Archimedean constant to eschew zero weights and $E_{d d}^{*}$ is the CCR efficiency of $D M U_{d}$ derived from the stochastic CCR model computed in phase (I).

Note that the following model could be considered in the phase (ii) as the stochastic benevolent version of cross-efficiency method

$$
\begin{aligned}
& \max \sum_{r=1}^{s} u_{r d} \sum_{j=1 . j \neq d}^{n} y_{r j} \\
& \text { s.t } \sum_{i=1}^{m} v_{i d} \sum_{j=1 . j \neq d}^{n} x_{i j}=1 \\
& \sum_{r=1}^{s} u_{r d} y_{r d}-E_{d d}^{*} \sum_{i=1}^{m} v_{i d} x_{i d}=0
\end{aligned}
$$




$$
\begin{aligned}
& \sum_{r=1}^{s} u_{r d} y_{r j}-\sum_{i=1}^{m} v_{i d} x_{i j}-\Phi^{-1}(\alpha) \delta_{j} \leq 0, \forall j \\
& u_{r d}, v_{i d} \geq \varepsilon \quad r=1, \ldots, s, i=1, \ldots, m \\
& \delta_{j}=\sum_{r=1}^{s} \sum_{k=1}^{s} u_{r} u_{k} \operatorname{Cov}\left(\tilde{y}_{r j}, \tilde{y}_{k j}\right)+\sum_{i=1}^{m} \sum_{l=1}^{m} v_{i} v_{l} \operatorname{Cov}\left(\tilde{x}_{i j}, \tilde{x}_{l j}\right) \\
& +2 \sum_{r=1}^{s} \sum_{i=1}^{m} u_{r} v_{i} \operatorname{Cov}\left(\tilde{y}_{r j}, \tilde{x}_{i j}\right), j=1, \ldots, n
\end{aligned}
$$

\section{Table 6.Aggressive cross-efficiency with stochastic data}

\begin{tabular}{|c|c|c|c|c|c|c|c|c|c|c|}
\hline \multicolumn{1}{|c|}{ Cross-Efficiency Scores } & \multicolumn{5}{c|}{ Optimal Weights } \\
\hline DMU & $\mathbf{( 1 )}$ & $\mathbf{( 2 )}$ & $\mathbf{( 3 )}$ & $\mathbf{( 4 )}$ & $\mathbf{( 5 )}$ & $\mathbf{( 6 )}$ & $\boldsymbol{v}_{\mathbf{1}}^{*}$ & $\boldsymbol{v}_{\mathbf{2}}^{*}$ & $\boldsymbol{u}_{\mathbf{1}}^{*}$ & $\boldsymbol{u}_{\mathbf{2}}^{*}$ \\
\hline $\mathbf{( 1 )}$ & 0.91 & 0.34 & 0.79 & 0.35 & 0.37 & 0.37 & 0.44 & 1.69 & 0.64 & 0.05 \\
\hline $\mathbf{( 2 )}$ & 0.74 & 1 & 0.44 & 0.79 & 0.78 & 0.76 & 0.05 & 1.14 & 0.05 & 0.44 \\
\hline $\mathbf{( 3 )}$ & 0.74 & 0.35 & 1 & 0.52 & 0.51 & 0.4 & 0.29 & 0.05 & 0.23 & 0.05 \\
\hline$(\mathbf{4})$ & 0.52 & 0.69 & 0.69 & 1 & 0.91 & 0.64 & 0.17 & 0.05 & 0.05 & 0.2 \\
\hline $\mathbf{( 5 )}$ & 0.9 & 1 & 0.72 & 1 & 1 & 0.89 & 0.1 & 0.54 & 0.08 & 0.34 \\
\hline$(\mathbf{6})$ & 0.99 & 1.06 & 0.79 & 1 & 1 & 0.9 & 0.14 & 0.77 & 0.15 & 0.46 \\
\hline$\widetilde{\boldsymbol{E}_{J}}$ & 0.8 & 0.74 & 0.74 & 0.78 & 0.76 & 0.66 & & & & \\
\hline
\end{tabular}

Table 7. Benevolent cross-efficiency with stochastic data

\begin{tabular}{|c|c|c|c|c|c|c|c|c|c|c|}
\hline \multicolumn{1}{|c|}{ Cross-Efficiency Scores } & \multicolumn{5}{c|}{ Optimal Weights } \\
\hline $\mathbf{D M U}$ & $\mathbf{( 1 )}$ & $\mathbf{( 2 )}$ & $\mathbf{( 3 )}$ & $\mathbf{( 4 )}$ & $\mathbf{( 5 )}$ & $\mathbf{( 6 )}$ & $\boldsymbol{v}_{\mathbf{1}}^{*}$ & $\boldsymbol{v}_{\mathbf{2}}^{*}$ & $\boldsymbol{u}_{\mathbf{1}}^{*}$ & $\boldsymbol{u}_{\mathbf{2}}^{*}$ \\
\hline $\mathbf{( 1 )}$ & 0.91 & 1.08 & 0.48 & 0.79 & 0.79 & 0.82 & 0.05 & 4.6 & 0.24 & 1.62 \\
\hline $\mathbf{( 2 )}$ & 1.01 & 1 & 0.99 & 1 & 1 & 0.90 & 0.15 & 0.59 & 0.19 & 0.35 \\
\hline $\mathbf{( 3 )}$ & 1.01 & 0.99 & 1 & 1 & 1 & $0 . .89$ & 0.13 & 0.5 & 0.16 & 0.29 \\
\hline$(\mathbf{4})$ & 1.02 & 0.93 & 1 & 1 & 0.98 & 0.85 & 0.09 & 0.28 & 0.11 & 0.17 \\
\hline $\mathbf{( 5 )}$ & 1.01 & 1 & 0.92 & 1 & 1 & 0.92 & 0.09 & 0.56 & 0.14 & 0.29 \\
\hline $\mathbf{( 6 )}$ & 1.01 & 1 & 0.98 & 1 & 1 & 0.9 & 0.16 & 0.7 & 0.21 & 0.4 \\
\hline$\widetilde{\boldsymbol{E}_{\boldsymbol{J}}}$ & 0.99 & 1 & 0.89 & 0.96 & 0.96 & 0.89 & & & & \\
\hline
\end{tabular}

Tables 6 and 7 shows the minimum and maximum efficiency scores while stochastic data are considered. Note that in this evaluation $\varepsilon=0.05$ is used considering $D M U_{1}$ as an example, we found that the cross-efficiency of $D M U_{1}$ is $0.99(0.72)$ in the maximum (minimum) mode. These calculations mean that the 
Cross-efficiency Evaluation in Data Envelopment Analysis with Stochastic Data: A Chance-constrained Programming Approach

computed efficiency has changed between 0.72 and 0.99 , so it is possible to use an efficiency interval [0.72. 0.99] for cross-efficiency score of this unit.

\section{Illustrative application}

To demonstrate the applicability of the suggested approach, we applied a data set including 13 open coastal Chinese cities and five Chinese special economic zones in 1989 which is taken from Zhu (2009). The data set includes three outputs and two inputs.

Inputs:

- Investment in fixed assets by state-owned enterprises $\left(x_{1}\right)$

- Foreign funds $\left(x_{2}\right)$

Outputs:

- Total industrial output value $\left(y_{1}\right)$

- Total value of retail sales $\left(y_{2}\right)$

- Handling capacity of coastal ports $\left(y_{3}\right)$

In this example, it is assumed that every index is a stochastic variable with normal distributions. The mean values of these variables are shown in the columns (2-6) of Table 8.

Inputs and outputs follow random data with a normal distribution and mean values are

follows: $x_{1} \sim N(\mu, 10), x_{2} \sim N(\mu, 15), y_{1} \sim N(\mu, 1), y_{2} \sim N(\mu, 10), y_{3} \sim N(\mu, 6)$ and $\quad$ a confidence interval of $95 \%$. To compute the results of models $(11,12)$, alpha was chosen to be at $\alpha=0.05$. Hence $\Phi^{-1}(\alpha)=1.645$ a computational issue is encountered in solving the above benevolent and aggressive models while calculating the cross efficiency scores. For both models, with deterministic data, indexes are shown in table 8 with $\bar{E}_{J}^{\text {max }}$ and $\bar{E}_{J}{ }^{\text {min }}$ and with stochastic data are shown in Table 8 with $\widetilde{\bar{E}}_{J}^{\max }$ and $\widetilde{\bar{E}}_{J}^{\min }$.

Considering $D M U_{17}$, the benevolent (aggressive) models of the deterministic data (Table 7) are $0.282(0.23)$, respectively. Furthermore, the benevolent (aggressive) models corresponding to the stochastic data (Table 8) are $0.317(0.222)$ for the same DMUs, respectively.This means that the maximum (minimum) likely cross efficiency scores of this DMU at the significance level $\alpha=0.05$ are in interval[0.222,0.317] while with deterministic data, their values fall in interval [0.23, 0.282]. Now, take $D M U_{1}$ and $D M U_{15}$ into consideration. The maximum cross efficiency of $D M U_{1}$ with stochastic data is 0.439 and that of $D M U_{15}$ is 0.19 . The minimum cross efficiency scores of these two $D M U s$ with stochastic data are 0.368 and 0.132 , respectively, for which we can consider an efficiency interval. As an instance, for $D M U_{1}$, the efficiency interval of [0.368, 0.439] can be considered and for $D M U_{15}$, the interval [0.132, 0.19] is possible. In this way, an important issue is the presence of multiple weights while stochastic data are being 
considered. Consider the same DMUs ( $D M U_{1}$ and $D M U_{15}$ ) for the definite state in Table 8. The benevolent cross efficiency of $D M U_{1}$ with deterministic data is 0.446 and that of $D M U_{15}$ is 0.166 . The aggressive cross efficiencies of these two DMUs with stochastic data are 0.378 and 0.139 , respectively, for which we can consider an efficiency interval. As an instance, the efficiency intervals of [0.378, $0.446]$ and $[0.139,0.166]$ can be considered for $D M U_{1}$ and $D M U_{15}$, respectively.

Table 8. Cross-efficiency score in Chinese cities with real data and random data and benevolent mode and Aggressive state

\begin{tabular}{|c|c|c|c|c|c|c|c|c|c|}
\hline & \multicolumn{5}{|c|}{ Data } & \multicolumn{2}{|c|}{ deterministic } & \multicolumn{2}{|c|}{ stochastic } \\
\hline DMU & $x_{1}$ & $x_{2}$ & $y_{1}$ & $y_{2}$ & $y_{3}$ & ${\overline{E_{J}}}^{\min }$ & ${\overline{E_{J}}}^{\max }$ & ${\widetilde{\boldsymbol{E}_{J}}}^{\min }$ & ${\widetilde{\bar{E}_{J}}}^{\max }$ \\
\hline (1) & 2874.800 & 16738.000 & 160.890 & 80800.000 & 5092.000 & 0.446 & 0.378 & 0.368 & 0.439 \\
\hline (2) & 946.300 & 691.000 & 21.140 & 18172.000 & 6563.000 & 1 & 0.916 & 0.913 & 1 \\
\hline (3) & 6854.000 & 43024.000 & 375.250 & 144530.000 & 2437.000 & 0.244 & 0.208 & 0.208 & 0.247 \\
\hline (4) & 2305.100 & 10815.000 & 176.680 & 70318.000 & 3145.000 & 0.453 & 0.388 & 0.386 & 0.457 \\
\hline (5) & 1010.300 & 2099.000 & 102.120 & 55419.000 & 1225.000 & 0.605 & 0.558 & 0.567 & 0.614 \\
\hline (6) & 282.300 & 757.000 & 59.170 & 27422.000 & 246.000 & 0.973 & 0.887 & 0.891 & 0.977 \\
\hline (7) & 17478.600 & 116900.000 & 1029.090 & 351390.000 & 14604.000 & 0.305 & 0.256 & 0.255 & 0.309 \\
\hline (8) & 661.800 & 2024.000 & 30.070 & 23550.000 & 1126.000 & 0.454 & 0.401 & 0.388 & 0.441 \\
\hline (9) & 1544.200 & 3218.000 & 160.580 & 59406.000 & 2230.000 & 0.57 & 0.518 & 0.538 & 0.597 \\
\hline (10) & 428.400 & 574.000 & 53.690 & 47504.000 & 430.000 & 0.884 & 0.874 & 0.8606 & 0.8605 \\
\hline (11) & 6228.100 & 29842.000 & 258.090 & 151356.000 & 4649.000 & 0.279 & 0.242 & 0.241 & 0.273 \\
\hline (12) & 697.700 & 3394.000 & 38.020 & 45336.000 & 1555.000 & 0.656 & 0.574 & 0.532 & 0.613 \\
\hline (13) & 106.400 & 367.000 & 7.070 & 8236.000 & 121.000 & 0.606 & 0.55 & 0.518 & 0.566 \\
\hline (14) & 4539.300 & 45809.000 & 116.460 & 56135.000 & 956.000 & 0.129 & 0.108 & 0.105 & 0.171 \\
\hline (15) & 957.800 & 16947.000 & 29.200 & 17554.000 & 231.000 & 0.166 & 0.139 & 0.132 & 0.19 \\
\hline (16) & 1209.200 & 15741.000 & 65.360 & 62341.000 & 618.000 & 0.386 & 0.334 & 0.308 & 0.431 \\
\hline (17) & 972.400 & 23822.000 & 54.520 & 25203.000 & 513.000 & 0.282 & 0.23 & 0.222 & 0.317 \\
\hline (18) & 2192.000 & 10943.000 & 25.240 & 40267.000 & 895.000 & 0.151 & 0.134 & 0.123 & 0.184 \\
\hline
\end{tabular}

Based on the results, the following ranking status in benevolent cross efficiency method is achieved for these data set: 
Cross-efficiency Evaluation in Data Envelopment Analysis with Stochastic Data: A Chance-constrained Programming Approach

- Deterministic data: $D M U_{2}>D M U_{6}>D M U_{10}>D M U_{12}>D M U_{13}>$ $D M U_{5}>D M U_{9}>D M U_{8}>D M U_{4}>D M U_{1}>D M U_{16}>D M U_{7}>$ $D M U_{17}>D M U_{11}>D M U_{3}>D M U_{15}>D M U_{18}>D M U_{14}$

- Stochastic data: $D M U_{2}>D M U_{6}>D M U_{10}>D M U_{5}>D M U_{12}>$ $D M U_{9}>D M U_{13}>D M U_{4}>D M U_{8}>D M U_{1}>D M U_{16}>D M U_{17}>$ $D M U_{7}>D M U_{11}>D M U_{3}>D M U_{15}>D M U_{18}>D M U_{14}$

Note that by allowing only $5 \%$ of the unsatisfied constraints for model (12), the provided rank position changed for some of the units. For example, $D M U_{5}$ achieved the 4th rank in stochastic case, while it was in the sixth place of performance in deterministic cross-efficiency evaluation. Only for $D M U_{1}, D M U_{2}, D M U_{3}, D M U_{10}, D M U_{11}, D M U_{14}, D M U_{15}, D M U_{16}$, and $D M U_{18}$ (two out of six), the stochastic rank is the same as deterministic rank. In other words, for these units, we are almost sure that they have a weak performance in comparison with other units.

Approximately $80 \%$ of the cross-efficiency interval obtained in exact data mode, are included in the computed interval for the case of stochastic data. Here $\alpha$, as a pre-specified acceptable risk, may also be employed for planning purposes. Therefore, it seems that the values of stochastic aggressive and benevolent cross efficiencies provide far better and more accurate prediction of efficiency status of the production systems in this sample.

\section{Conclusion}

This paper develops a new approach in DEA framework for calculating cross-efficiency scores in the presence of multiple optimal weights and stochastic inputs/outputs data. Estimating cross-efficiency scores as well as introducing some secondary goals are considered using the chance-constrained programming approach. Some new chance-constrained DEA models are suggested and the associated deterministic equivalent models are then introduced for computation purpose. It is shown that the issue of existence multiple optimal weights is still happens when using stochastic data in cross efficiency evaluation. New introduced stochastic version of benevolent and aggressive secondary goals is then used in cross efficiency evaluation of some numerical examples. The results demonstrate that the new stochastic cross-efficiency method provides more reliable crossefficiency scores for ranking production units. Further research could be conducted in dealing with the stochastic data in DEA framework, especially in providing some new cross-efficiency secondary goals.

\section{Appendix}

Assume all the variables have normal distribution with a known mean and variance (Rao 2009).

Thus, the objective function also has a normal distribution with a mentioned mean and variance as follows: 
Somayeh Rahmani, Reza Kazemi Matin, Mohsen Khunsiavash ,Zohreh Moghadas

$\bar{f}=\mu_{f}=\sum u_{r d} \tilde{y}_{r d}, \operatorname{Var}\left(\mu_{f}\right)=Y^{T} V Y$

Note that $V$ represents the variance-covariance matrix of $\tilde{y}_{n} \mathrm{~s}$ as:

$V=\left[\begin{array}{ccc}\operatorname{Var}\left(\tilde{y}_{1}\right) & \operatorname{Cov}\left(\tilde{y}_{1}, \tilde{y}_{2}\right) \ldots & \operatorname{Cov}\left(\tilde{y}_{1}, \tilde{y}_{n}\right) \\ \operatorname{Cov}\left(\tilde{y}_{2}, \tilde{y}_{1}\right) & \operatorname{Var}\left(\tilde{y}_{2}\right) \ldots & \operatorname{Cov}\left(\tilde{y}_{2}, \tilde{y}_{n}\right) \\ \ldots & \ldots & \ldots \\ \operatorname{Cov}\left(\tilde{y}_{n}, \tilde{y}_{1}\right) & \operatorname{Cov}\left(\tilde{y}_{n}, \tilde{y}_{2}\right) & \operatorname{Var}\left(\tilde{y}_{n}\right)\end{array}\right]$

It is possible to consider a new objective function in the following form.

$F(\underline{\tilde{x}})=k_{1} \bar{f}+k_{2} \sqrt{\operatorname{var}(f)}$, where $k_{1}$ and $k_{2}$ are non-negative constants, whose values indicate the importance of $\bar{f}$ and the standard deviation of $f$ in the maximization problem.

if $k_{2}=0$ then $f(\underline{\tilde{x}})=\bar{f}$, if $k_{1}=0$ then $f(\underline{\tilde{x}})=\sqrt{\operatorname{var}(f)}$

For simplification of notation, we rewrite the second constraint in Model (3.1) as follows:

$p\left[h_{j} \leq 0\right] \geq 1-\alpha, h_{j}=\sum_{r=1}^{s} u_{r d} \tilde{y}_{r j}-\sum_{i=1}^{m} v_{i d} \tilde{x}_{i j}$

As we know $\quad v\left(h_{j}\right)=\tilde{Y}^{T} v_{j} \tilde{Y}$ in which $V$ is the variance. Therefore:

$$
\mathrm{V}_{\mathrm{j}}=\left[\begin{array}{ccc}
\operatorname{Var}\left(\tilde{y}_{r 1}\right) & \operatorname{Cov}\left(\tilde{y}_{r 1}, \tilde{y}_{r 2}\right) \ldots & \operatorname{Cov}\left(\tilde{y}_{r 1}, \tilde{x}_{i d}\right) \\
\operatorname{Cov}\left(\tilde{y}_{r 2}, \tilde{y}_{r 1}\right) & \operatorname{Var}\left(\tilde{y}_{r 2}\right) \ldots & \operatorname{Cov}\left(\tilde{y}_{r 2}, \tilde{x}_{i d}\right) \\
\ldots & \ldots & \ldots \\
\operatorname{Cov}\left(\tilde{y}_{i d}, \tilde{y}_{i 1}\right) & \operatorname{Cov}\left(\tilde{y}_{i d}, \tilde{y}_{i 2}\right) & \operatorname{Var}\left(\tilde{x}_{i d}\right)
\end{array}\right] \text { and } y=\left[\begin{array}{c}
\tilde{y}_{1} \\
\tilde{y}_{2} \\
\ldots \\
\tilde{y}_{n}
\end{array}\right]
$$

Thus:

$$
\begin{array}{r}
\operatorname{Var}\left(h_{j}\right)=\sum_{r=1}^{s}\left(u_{r d}^{2} \operatorname{Var}\left(\tilde{y}_{r j}\right)+\sum_{l=1}^{s} u_{r d} u_{l d} \operatorname{Cov}\left(\tilde{y}_{r j}, \tilde{y}_{l j}\right)\right)+\sum_{i=1}^{m}\left(v_{i d}^{2} \operatorname{Var}\left(\tilde{x}_{i j}\right)\right) \\
\left.+\sum_{e=1}^{m} v_{i d} v_{e d} \operatorname{Cov}\left(\tilde{x}_{i j}, \tilde{x}_{e j}\right)\right)-2 \operatorname{Cov}\left(\sum_{r=1}^{s} u_{r d} \tilde{y}_{r j}, \sum_{i=1}^{m} v_{i d} \tilde{x}_{i j}\right)
\end{array}
$$

Consider $h_{j}$ to be normally distributed, and $h_{j} \sim N\left(\bar{h}_{j}, \operatorname{Var}\left(h_{j}\right)\right)$. According to the constraint, we will have:

$p\left[\frac{h_{j}-\overline{h_{j}}}{\sqrt{\operatorname{Var}\left(h_{j}\right)}} \leq \frac{-h_{j}}{\sqrt{\operatorname{Var}\left(h_{j}\right)}}\right] \geq 1-\alpha, \quad$ If $\Phi\left(s_{j}\right)=1-\alpha$ then $\Phi\left(\frac{-h_{j}}{\sqrt{\operatorname{Var}\left(h_{j}\right)}}\right) \geq$ $\Phi\left(s_{j}\right) ; \quad j=1 \ldots n$

According to the above inequality, we may write: 
Cross-efficiency Evaluation in Data Envelopment Analysis with Stochastic Data: A Chance-constrained Programming Approach

$\left(\frac{-h_{j}}{\sqrt{\operatorname{Var}\left(h_{j}\right)}}\right) \geq s_{i}$, Then $s_{j}=\Phi^{-1}(1-\alpha)$ or equivalently $\bar{h}_{j}+s_{j} \sqrt{\operatorname{Var}\left(h_{j}\right)} \leq 0 \quad j=$

$1 \ldots n, x_{j} \geq 0$. This leads to $\bar{h}_{j}+\Phi^{-1}(1-\alpha) \sqrt{\operatorname{Var}\left(h_{j}\right)} \leq 0$.

\section{REFERENCES}

[1]Alcaraz, J., Ramon, R., Ruiz, J.\&Sirvent, I.(2013), Ranking Range in CrossEfficiency Evaluation. Journal of Productivity Analysis, 516-521;

[2] Anderson, T.R., Hollings Worth, K.B.\& Inman, L. B.(2002), The Fixed Weighting Nature of a Cross Evaluation Model. Journal of Productivity Analysis, 18, 249-255;

[3]Azadi, M.\&Saen, R.F.(2011),A New Chance-Constrained Data Envelopment Analysis for Selecting Third-Party Reverse Logistics Providers in the Existence of Dual-Role Factors. Expert Systems with applications. Journal of Elsevier,38(10), 12231-12236;

[4] Charnes, A., Cooper, W.W.\& Rhodes, E.(1978),Measuring the Efficiency of Decision Making Units.European journal of operationalResearch, 2, 429-444;

[5]Chen, L.\&Jia, G.(2016),Environmental Efficiency Analysis of China's

Regional Industry a Data Envelopment Analysis (DEA) Based Approach. Journal of Cleaner Production, Http dx.doi.org/10.1016/j.jclepro, 01-045;

[6] Cooper, W.W., Honghui, D., Huang, Z.\& Li, S.X.(2004), Chance

Constrained Programming Approaches to Congestion in Stochastic Data

Envelopment Analysis.European Journal of Operational Research,155 (2),487-501;

[7]Cooper, W.W., Deng, H., Huang, Z.\& Li, S.X. (2002),ChanceConstrained

Programming Approaches to Technical Efficiencies and Inefficiencies in

Stochastic Data Envelopment Analysis. Journal of the Operational Research Society, 53, 1347-1356;

[8] Doyle, J.R., Green, R.(1994), Efficiency and Cross-efficiency in Data Envelopment Analysis: Derivatives Meaning and Uses.Journal of Operational Research society, 45(5), 567-578;

[9]Emrouznejad, A.\& Yang, G. L. (2018), A Survey and Analysis of the first 40 Years of Scholarly Literature in DEA.Socio-Economic PlanningSciences, 61, 4-8; [10] Hiroshi, M.\&Seiford, L.M.(1999),Characteristics on Stochastic DEA Efficiency.Journal of the Operations Research Society ofJapan, 42 (4), 389-404; [11] Kao, C.\& Liu, S.T.(2009),Stochastic Data Envelopment Analysis in Measuring the Efficiency of Taiwan Commercial Banks. European Journal of Operational Research, 196(1), 312-322;

[12] Khodabakhshi, M.(2009),Estimating Most Productive Scale Size with Stochastic Data in Data Envelopment Analysis. Economic Modelling, doi: 10. 1016.j. econmod.2009.03.002;

[13] Kuosmanen, T.\& Johnson, A.(2010),Data Envelopment Analysis as Nonparametric Least Square Regression. Operations Research, 58, 149-160; 
Somayeh Rahmani, Reza Kazemi Matin, Mohsen Khunsiavash ,Zohreh Moghadas

[14] Kuosmanen, T.\&Kortelainen, M. (2012),Stochastic Non-Smooth Envelopment of Data: Semi-Parametric Frontier Estimation Subject to Shape Constraints.Journal of Productivity Analysis, 38,11-28;

[15] Kuosmanen, T., Johnson, A.\&Saastamoinen, A.(2015),Stochastic Nonparametric Approach to Efficiency Analysis. A United Framework. In: Zhu. J. (Ed.) Data Envelopment Analysis.Springer US, 221, 191-224;

[16] Li, Y., Lei, X., Dai, Q.\& Liang, L.(2015),Performance Evaluation of Participating Nations at the 2012 London Summer Olympics by a Two-Stage Data Envelopment Analysis.European Journal of Operational Research, 243 (3), 964-973;

[17] Li, H., Chen, C., Cook, W. D., Zhang, J.\& Zhu, J. (2018),Two-stage

Network DEA: Who is the Leader.Omega, 74, 15-19;

[18] Lim, S., Oh, K.W.\& Zhu, J.(2014),Use of DEA Cross-Efficiency

Evaluation in Portfolio Selection: An Application to Korean Stock

Market.European Journal of Operational Research, 36(1), 361-368;

[19] Mardani,A.,Zavadskas, E. K.,Streimikiene, D.,Jusoh, A.\&Khoshnoudi,M. (2016), A Comprehensive Review of Data Envelopment Analysis (DEA) Approach in Energy Efficiency. Renewable and Sustainable Energy Reviews, 70, 1298-1322; [20] Oral, M., Amin, G.\&Oukil, A.(2015),Cross-efficiency in DEA a Maximum Resonated Appreciative Model.Measurement, 12.006,159-167;

[21]Rao, S. S,John Wiley \& Sons, Inc(2009),Stochastic

Programming,Engineering Optimization Theory and Practice; John Wiley \& Sons, Inc., Hoboken, New Jersey, simultaneously in Canada, chapter 11;

[22] Sexton, T.R., Silkman, R.H., Hogan, A.J.(1986),Data Envelopment Analysis: Critique and Extensions in Silkman.R.H.(Ed.) Measuring Efficiency: AN Assessment of Data Envelopment Analysis. Jossey-Bass. San Francisco, 32,73105 ;

[23] Soltanifar, M., Shahghobadi, S.(2013),Selecting a Benevolent Secondary Goal Model in Data an Envelopment Analysis Cross-Efficiency Evaluation by a Voting.Socio-Economic Planning Sciences, 159-167;

[24] Wu, J., Sun, J. S.\& Liang, L.(2012),Cross Efficiency Evaluation Method Based on Weight-balanced Data Envelopment Analysis Model. Computers and Industrial Engineering, 63, 513-519;

[25]Wu, J., Chu, J.F., Sun, J.S. \& Zhu, Q.Y. (2016), DEA Cross Efficiency

Evaluation Based on Pareto Improvement. European Journal of Operational Research,248 (2),571-579. 